\title{
The Future of Artificial Intelligence for Alzheimer's Disease Diagnostics
}

\author{
Robert Logan1,2, Sabrina S. Zerbey², Sean J. Miller ${ }^{1}$ \\ ${ }^{1}$ Pluripotent Diagnostics, Molecular Medicine Research Institute, Sunnyvale, CA, USA \\ ${ }^{2}$ Department of Biology, Eastern Nazarene College, Quincy, MA, USA \\ Email: DrMiller@PluriPotentDiagnostics.com
}

How to cite this paper: Logan, R., Zerbey, S.S. and Miller, S.J. (2021) The Future of Artificial Intelligence for Alzheimer's Disease Diagnostics. Advances in Alzheimer's Disease, 10, 53-59.

https://doi.org/10.4236/aad.2021.104005

Received: October 1, 2021

Accepted: December 13, 2021

Published: December 16, 2021

Copyright $\odot 2021$ by author(s) and Scientific Research Publishing Inc. This work is licensed under the Creative Commons Attribution International License (CC BY 4.0).

http://creativecommons.org/licenses/by/4.0/

\begin{abstract}
Alzheimer's disease $(\mathrm{AD})$ is a leading cause of death, yet there is no disease-modifying drug therapy currently available. It is critical to establish a diagnosis of $\mathrm{AD}$ before clinical system onset so that drug therapies can start earlier. Unfortunately, this is not the current standard practice. Artificial intelligence (AI) holds tremendous promise for identifying $\mathrm{AD}$ related structural changes in brain scan images. This paper discusses the recent applications and potential future directions for $\mathrm{AI}$ in $\mathrm{AD}$ diagnostics. Annual brain scanning and computer vision-assisted early diagnosis is encouraged, so that disease-modifying drug therapy could begin earlier in the progressive pathology.
\end{abstract}

\section{Keywords}

Artificial Intelligence, Convolutional Neural Network, Disease Detection, Neurodegeneration, Alzheimer's Disease

\section{Introduction}

Rapid technological advancements in various fields have recently ushered in a biomedical revolution. Modern life scientists now rely on interdisciplinary approaches to tackle what was previously impossible. Artificial intelligence is one of the most promising cutting-edge tools being perfected for clinical use, especially for diagnostic applications. In the recently published article, "Deep Convolutional Neural Networks with Ensemble Learning and Generative Adversarial Networks for Alzheimer's Disease Image Data Classification", the authors provide a comprehensive overview of the strategies and benefits of incorporating artificial intelligence (AI) into Alzheimer's disease (AD) detection from magnetic 
resonance imaging (MRI) in order to achieve extremely high accuracy and precision in determining the presence of disease [1].

Biomedical data is being generated at an unprecedented pace. Biomedical scientists and clinicians alike need to use high-performance computing, bioinformatics and cloud storage to efficiently and accurately analyze these data in a meaningful way. The modern problems of working with large datasets require the modern solution of AI, specifically utilizing the deep learning subdiscipline of AI. The general interest surrounding AI, which includes machine learning and deep learning, has experienced a meteoric rise in recent history due to the promise it holds in revolutionizing healthcare [2]. However, it has been clarified that "AI won't replace radiologists, but radiologists who use AI will replace radiologists who don't" [3].

There is a pressing need to establish an earlier diagnosis for those with neurodegenerative diseases. By the time a diagnosis is made, the extent of damage to the central nervous system is beyond repair. It has been shown repeatedly that the best defense against neurodegeneration is an early offense, which is only made possible through an early diagnosis [4]. Due to the large extent that $\mathrm{AD}$ occurs in the population, the tremendous societal burden of $\mathrm{AD}$, and the long asymptomatic preclinical period $\mathrm{AD}$, there is significant potential that an early diagnosis could be critical for turning the tide of the disease [5].

At the present, an $\mathrm{AD}$ diagnosis is established by the clinical presentation of cognitive and memory deficits in addition to the histopathological presence of a mixed proteinopathy. The biomarkers necessary for an $\mathrm{AD}$ diagnosis consist of $\beta$-amyloid plaques and neurofibrillary tangles composed of hyperphosphorylated tau [6] [7]. The incorporation of histological markers through either imaging, blood or cerebral spinal fluid (CSF) analysis, is useful in clearly differentiating between mild cognitive impairment $(\mathrm{MCI})$, various stages of $\mathrm{AD}$, or other forms of dementia, such as dementia with Lewy bodies or frontotemporal dementia [8] [9] [10].

Non-invasive or minimally invasive imaging has become a valuable asset in the development of $\mathrm{AD}$ diagnosis. For instance, PET scans for $\beta$-amyloid plaques require the injection of a specialized radiolabeled tracer, such as florbetapir [11]. PET scans for AD diagnostics have been shown to have at least $96 \%$ sensitivity and $100 \%$ specificity, even for milder forms of the disease [7]. Despite being FDA approved for many years and offering high diagnostic accuracy, PET imaging has not become standard clinical practice due to high costs and patient concerns over the use of radiolabeled tracers. MRI scans, however, only use magnet and radio waves for imaging. Therefore, MRIs do not use X-rays like those used for computerized tomography (CT) and do not use radiolabeled tracers like those used for PET scans. However, MRIs are impractical for some patients who cannot be exposed to strong magnet waves, like those with a heart pacemaker or an aneurysm clip. MRIs can accurately detect cortical atrophy patterns, ventricle enlargements, $\beta$-amyloid plaques and neurofibrillary tangle presence and density among AD patients [12] [13]. Furthermore, brain imaging offers a less invasive 
alternative to histopathological analysis than a lumbar spinal tap for CSF collection. It is the opinion of the authors that routine brain imaging should become a part of routine medical care, just as mammograms and prostate cancer checks have become (Figure 1). However, the risk/benefit analysis of modality type and brain scan frequency should be established at the direction of a physician to reduce any unnecessary exposure to radiation. Finally, in order to have brain imaging become established as a part of routine screenings, there also needs to be an established standardization of image interpretation. This is where deep learning becomes valuable.

\section{Convolutional Neural Networks}

Convolutional neural networks (CNNs) are a widely used computer vision tool for image classification. Briefly, CNNs receive an image input such as an MRI to create the input layer, which then feeds into the convolution layers followed by pooling layers. There may be many convolution and pooling layers in a $\mathrm{CNN}$ to extract low-level image information in the early stages of the network and to reduce dimensionality until high-level final details of the image are extracted and learned according to weights and biases. Finally, a fully connected network of layers is built. Critical to the architecture of the CNN is the kernel, which provides learnability and produces a feature map. The final output is then sent through a non-linear activation function, such as a logistic or hyperbolic tangent function. A benefit of the CNN is that it is able to quickly learn the relevant features and patterns of an MRI without requiring flattening or prior knowledge of important features and their manual extraction. Furthermore, CNN is able to perform deep multiclassification analysis to distinguish between patients who are cognitively normal (CN), have mild cognitive impairment (MCI), or AD [14]. The commonly used GoogLeNet, ResNet-18 and ResNet-152 CNN architectures have been shown to have over $98 \%$ accuracy in classifying $\mathrm{CN}, \mathrm{MCI}$, late MCI and AD on patient MRI data [15] [16].

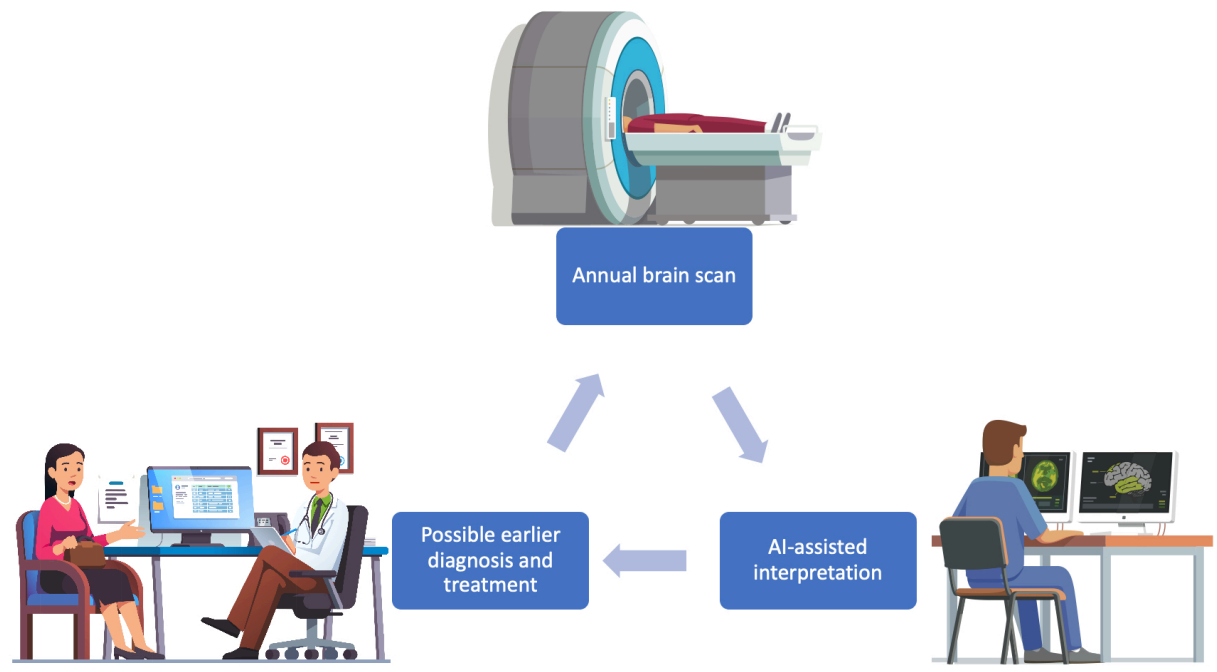

Figure 1. Proposed strategy for establishing earlier AD diagnosis. 


\section{Generative Adversarial Networks}

Generative adversarial networks (GAN) are comprised of a generative neural network paired with a discriminative neural network and both networks antagonize each other. The role of the generative network is to produce an example image based on prior input. The role of the discriminator is to assess the output of the generative network to see if the example images match well to the images used in training. Throughout this process, the generative neural network learns to build better matching images until the discriminator is satisfied. GANs are commonly used for image data augmentation, which is beneficial in image-based AD diagnosis. For example, GANs can be used to help enhance an MRI image or to actually predict whole brain structural changes throughout time. Therefore, GANs can hold tremendous promise in playing a critical role in establishing an AD diagnosis. Variation in magnet strength can impact the quality of MRI images. Common quality classifications include the base quality $1.5-\mathrm{T}$ and the superior 3-T scans. 3-T images reduce the signal-to-noise ratio of $1.5-\mathrm{T}$ by half. GANs have been used to take 1.5-T quality input images and convert them into 3-T quality images, which led to better AD classification [17]. Multimodal assessment, such as combining MRI and PET scans, can increase accuracy in diagnostics over single model assessment alone. However, PET images are not always available. It has recently been shown that GANs can be used to build PET scans to compliment MRI scans from the same patient with high accuracy [18].

\section{Ensemble Learning}

Ensemble learning (EL) makes use of several learning algorithms simultaneously for the purpose of completing the same image identification task. EL yields a better predictive quality than the individual learning algorithms would otherwise in isolation because the multiple outcomes can be combined, hence the use of the word "ensembled" in its title. EL tends to result in higher accuracy, lower bias errors, lower variance errors, and avoids overfitting. Therefore, it is useful when a single learning algorithm of interest overfits the data. EL has recently been used to classify patient brain PET image data into CN or AD (91\% accuracy) and mild MCI or severe MCI (86\% accuracy) [19]. DTE is an even more recent novel EL model, which was able to achieve classification accuracy of $99.09 \%$ for discriminated between $\mathrm{CN}$ and $\mathrm{AD}$ and $98.71 \%$ for discrimination between $\mathrm{MCI}$ and $\mathrm{AD}$ based on the ADNI dataset [20]. Another recently developed EL tool called DELearning incorporates multilayer deep learning with EL to classify AD with more precision, recall, accuracy, and F1-measure score than six other popular EL methods including AdaBoost, Bagging, LogitBoost, Random Forest, Stacking and Vote [21]. Additionally, a stack of GoogleLeNet, ResNet and DenseNet CNNs was recently used in an EL algorithm for multimodal image classification [22]. Both MRI and PET images were then combined with AdaBoost for an accuracy of $99.27 \%$ for identifying $\mathrm{CN}$ verses $\mathrm{AD}, 92.57 \%$ for identifying $\mathrm{AD}$ verses $\mathrm{MCI}$, and $90.35 \%$ for identifying $\mathrm{MCI}$ verses $\mathrm{CN}$ patients [22]. 


\section{Conclusion}

Logan and colleagues offer a compelling argument for routine computer vision assisted assessment of central nervous system (CNS) changes that are indicative of neurodegeneration onset before clinical symptoms present [1]. Synonymous with routine screening for prostate cancer, breast cancer, or high blood pressure, routine brain imaging with the assistance of deep learning algorithm interpretation is necessary for the establishment of early diagnosis and more effective therapeutics for AD. Deep learning appears to be very promising at revolutionizing healthcare practice, but it still has some maturing to do. For example, when AI was applied in the clinic for COVID-19 diagnostics based on chest radiographs and CT scans, it failed miserably [23]. In order to advance the cause of AI in diagnostics, better training datasets are needed, external validation is needed, and better quality publicly available documentation is needed to facilitate reproducibility [23]. Despite the initial growing pains of getting AI successfully into the clinic, the efforts are well worth it. The new dawn of deep learning in radiology and the hope it brings to physicians, scientists and the families of patients will break over the horizon very soon.

\section{Conflicts of Interest}

The authors declare no conflicts of interest regarding the publication of this paper.

\section{References}

[1] Logan, R., et al. (2021) Deep Convolutional Neural Networks with Ensemble Learning and Generative Adversarial Networks for Alzheimer's Disease Image Data Classification. Frontiers in Aging Neuroscience, 13, Article ID: 720226. https://doi.org/10.3389/fnagi.2021.720226

[2] Reardon, S. (2019) Rise of Robot Radiologists. Nature, 576, S54-S58. https://doi.org/10.1038/d41586-019-03847-Z

[3] Langlotz, C.P. (2019) Will Artificial Intelligence Replace Radiologists? Radiology. Artificial Intelligence, 1, e190058. https://doi.org/10.1148/ryai.2019190058

[4] van Oostveen, W.M. and de Lange, E.C.M. (2021) Imaging Techniques in Alzheimer's Disease: A Review of Applications in Early Diagnosis and Longitudinal Monitoring. International Journal of Molecular Sciences, 22, Article No. 2110. https://doi.org/10.3390/ijms22042110

[5] Aisen, P.S., et al. (2017) On the path to 2025: Understanding the Alzheimer's Disease Continuum. Alzheimer's Research \& Therapy, 9, Article No. 60.

[6] Deture, M.A. and Dickson, D.W. (2019) The Neuropathological Diagnosis of Alzheimer's Disease. Molecular Neurodegeneration, 14, Article No. 32. https://doi.org/10.1186/s13024-019-0333-5

[7] Weller, J. and Budson, A. (2018) Current Understanding of Alzheimer's Disease Diagnosis and Treatment. F1000Research, 7, 9 p. https://doi.org/10.12688/f1000research.14506.1

[8] Albert, M.S., et al. (2011) The Diagnosis of Mild Cognitive Impairment due to Alzheimer's Disease: Recommendations from the National Institute on Aging-Alzheimer's 
Association Workgroups on Diagnostic Guidelines for Alzheimer's Disease. Alzheimer's \& Dementia, 7, 270-279.

[9] McKhann, G.M., et al. (2011) The Diagnosis of Dementia Due to Alzheimer's Disease: Recommendations from the National Institute on Aging-Alzheimer's Association Workgroups on Diagnostic Guidelines for Alzheimer's Disease. Alzheimer's \& Dementia, 7, 263-269. https://doi.org/10.1016/j.jalz.2011.03.005

[10] Sperling, R.A., et al. (2011) Toward Defining the Preclinical Stages of Alzheimer's Disease: Recommendations from the National Institute on Aging-Alzheimer's Association Workgroups on Diagnostic Guidelines for Alzheimer's Disease. Alzheimer's \& Dementia, 7, 280-292.

[11] Saint-Aubert, L., et al. (2013) Cortical Florbetapir-PET Amyloid Load in Prodromal Alzheimer's Disease Patients. EJNMMI Research, 3, Article No. 43.

https://doi.org/10.1186/2191-219X-3-43

[12] Josephs, K.A., et al. (2013) Quantitative Neurofibrillary Tangle Density and Brain Volumetric MRI Analyses in Alzheimer's Disease Presenting as Logopenic Progressive Aphasia. Brain and Language, 127, 127-134.

https://doi.org/10.1016/j.bandl.2013.02.003

[13] Gong, N.J., Dibb, R., Bulk, M., van der Weerd, L. and Liu, C. (2019) Imaging Beta Amyloid Aggregation and Iron Accumulation in Alzheimer's Disease Using Quantitative Susceptibility Mapping MRI. NeuroImage, 191, 176-185.

https://doi.org/10.1016/j.neuroimage.2019.02.019

[14] Wang, S.H., Phillips, P., Sui, Y., Liu, B., Yang, M. and Cheng, H. (2018) Classification of Alzheimer's Disease Based on Eight-Layer Convolutional Neural Network with Leaky Rectified Linear Unit and Max Pooling. Journal of Medical Systems, 42, Article No. 85. https://doi.org/10.1007/s10916-018-0932-7

[15] Prakash, D., Madusanka, N., Bhattacharjee, S., Park, H.G., Kim, C.H. and Choi, H.K. (2019) A Comparative Study of Alzheimer's Disease Classification Using Multiple Transfer Learning Models. Journal of Multimedia Information System, 6, 209-216. https://doi.org/10.33851/JMIS.2019.6.4.209

[16] Farooq, A., Anwar, S.M., Awais, M. and Rehman, S. (2017) A Deep CNN Based Multi-Class Classification of Alzheimer's Disease Using MRI. 2017 IEEE International Conference on Imaging Systems and Techniques (IST), Beijing, 18-20 October 2017, 1-6. https://doi.org/10.1109/IST.2017.8261460

[17] Zhou, X., et al. (2020) Enhancing MR Imaging Driven Alzheimer's Disease Classification Performance Using Generative Adversarial Learning. MedRxiv. https://doi.org/10.1101/2020.07.22.20159814

[18] Lin, W., et al. (2021) Bidirectional Mapping of Brain MRI and PET With 3D Reversible GAN for the Diagnosis of Alzheimer's Disease. Frontiers in Neuroscience, 15, Article ID: 646013. https://doi.org/10.3389/fnins.2021.646013

[19] Zheng, C., Xia, Y., Chen, Y., Yin, X. and Zhang, Y. (2018) Early Diagnosis of Alzheimer's Disease by Ensemble Deep Learning Using FDG-PET. In: Peng, Y., Yu, K., Lu, J. and Jiang, X., Eds., Intelligence Science and Big Data Engineering, Springer, Cham, 614-622. https://doi.org/10.1007/978-3-030-02698-1 53

[20] Tanveer, M., Rashid, A.H., Ganaie, M.A., Reza, M., Razzak, I. and Hua, K.L. (2021) Classification of Alzheimer's Disease Using Ensemble of Deep Neural Networks Trained through Transfer Learning. IEEE Journal of Biomedical and Health Informatics (Early Access), 25 May 2021, 1. https://doi.org/10.1109/JBHI.2021.3083274

[21] An, N., Ding, H., Yang, J., Au, R. and Ang, T.F.A. (2020) Deep Ensemble Learning for Alzheimer's Disease Classification. Journal of Biomedical Informatics, 105, Ar- 
ticle ID: 103411. https://doi.org/10.1016/j.jbi.2020.103411

[22] Fang, X., Liu, Z. and Xu, M. (2020) Ensemble of Deep Convolutional Neural Networks Based Multi-Modality Images for Alzheimer's Disease Diagnosis. IET Image Processing, 14, 318-326. https://doi.org/10.1049/iet-ipr.2019.0617

[23] Roberts, M., et al. (2021) Common Pitfalls and Recommendations for Using Machine Learning to Detect and Prognosticate for COVID-19 Using Chest Radiographs and CT Scans. Nature Machine Intelligence, 3, 199-217.

https://doi.org/10.1038/s42256-021-00307-0 